\title{
Twinning in copper deformed at high strain rates
}

\author{
S CRONJE*, R E KROON, W D ROOS and J H NEETHLING ${ }^{\dagger}$ \\ Department of Physics, University of Free State, Bloemfontein, South Africa \\ $\nmid$ Department of Physics, Nelson Mandela Metropolitan University, Port Elizabeth, South Africa
}

MS received 25 February 2010; revised 13 August 2012

\begin{abstract}
Copper samples having varying microstructures were deformed at high strain rates using a splitHopkinson pressure bar. Transmission electron microscopy results show deformation twins present in samples that were both annealed and strained, whereas samples that were annealed and left unstrained, as well as samples that were unannealed and strained, are devoid of these twins. These deformation twins occurred at deformation conditions less extreme than previously predicted.
\end{abstract}

Keywords. Copper; twinning; strain rates; grain sizes.

\section{Introduction}

The strength and ductility of metals is a vast and important research area in which certain trends are well known, but where it is difficult to predict results with a high level of certainty, especially under extreme conditions e.g. high strain rates and very small grain sizes. It is generally known that metals with a face-centred cubic crystal structure, such as copper, are ductile and deform plastically due to dislocation slip on (111) planes in the $\langle 110\rangle$ directions. An alternative deformation mechanism, namely the formation of twins, usually occurs in body-centred cubic crystal structure metals at low (room) temperature and in hexagonal close packed structured metals (Cullity 1978). However, deformation twinning of face-centred cubic crystal structure metals is known to occur at very high strain rates (Meyers et al 2001). In this study, the microstructure of copper samples deformed at high strain rates (700 and $1400 \mathrm{~s}^{-1}$ ) were investigated using both scanning electron microscopy (SEM) and transmission electron microscopy (TEM).

Twinned crystals consist of two parts symmetrically related to one another either by rotation or reflection. Twins can originate during annealing or deformation. It is important to note that the crystal orientation on either side of a twin boundary is not the same, which gives rise to additional diffraction spots (twin spots) in an electron diffraction image. For face-centred cubic crystal metals like copper, the relationship between these two parts as described above can be obtained by a reflection across the (111) plane normal to the twin axis. When metallographic analysis of a metal sample takes place, annealing twins may appear in two forms. In figure 1(a) one part (B) of the grain is twinned with respect to the other part (A). The two parts are connected on the composition plane (111) which makes a line trace on the plane

\footnotetext{
*Author for correspondence (CronjeS@ufs.ac.za)
}

of polish. The more common variation is seen in figure 1(b). The grain shows three parts. Parts A1 and A2 are of identical orientation, but are separated by part B known as a twin band. This twin band is twinned with respect to A1 and A2 (Cullity 1978).

These twins are the result of a change in the normal growth mechanism. Suppose during grain growth, a grain boundary is parallel to the (111) plane and is advancing in a direction normal to this boundary (111). During this advancing stage, atoms leave the lattice of the consumed grain and join that of the growing grain. These layers are added parallel to the (111) plane in the sequence $\mathrm{ABCABC}$ for a face-centred cubic crystal. A twin will occur if an anomaly occurs in this layering sequence. If this sequence is altered to CBACBA, the crystal so formed will still be face-centred cubic, but will be twinned with respect to the parent crystal. If a similar occurrence takes place at a later stage, a crystal with the original orientation will start growing, thus forming a twin band.

\section{Experimental}

Cylindrical high purity copper samples of about $5 \mathrm{~mm}$ in both length and diameter were extracted out of a manufactured article using electric discharge machining (EDM). This was done to minimize stress and heating effects on the sample microstructure and composition. Some samples were left unannealed, while others were annealed at 300 or $500{ }^{\circ} \mathrm{C}$ for $30 \mathrm{~min}$, in order to obtain a variety of starting microstructures. Samples at room temperature were then strained at two possible high strain rates (700 and $1400 \mathrm{~s}^{-1}$ ) using a split-Hopkinson pressure bar. For a full description of this apparatus see Gray (2000) and Marais et al (2004).

The resulting microstructures of these strained as well as unstrained samples were then studied using both scanning electron microscopy (SEM) and transmission electron 

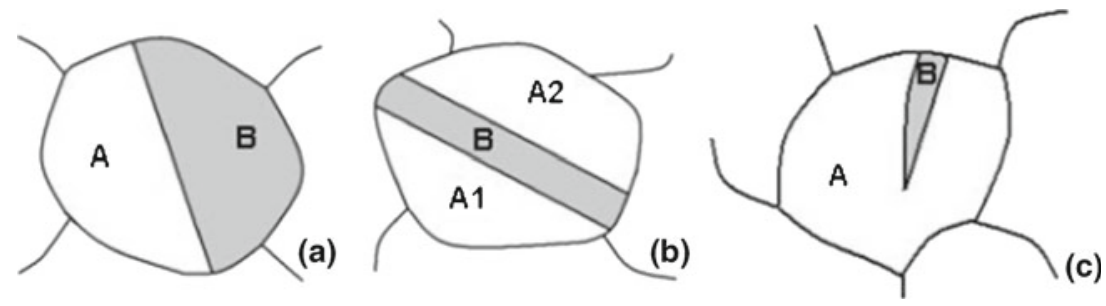

Figure 1. (a) Annealing twin of type I, (b) annealing twin of type II and (c) deformation twin. (adapted from Cullity 1978, p 60).
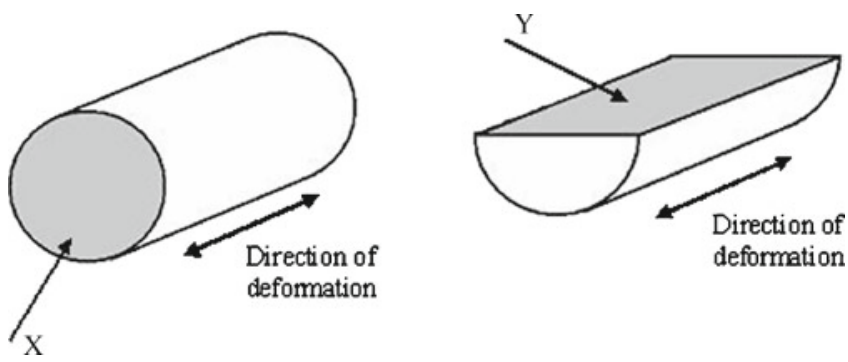

Figure 2. Surfaces of samples used to determine average grain size.

microscopy (TEM). Sample surfaces to be viewed using SEM were polished before etching. Polishing was performed using various grades of sandpaper followed by polishing with diamond suspension. The samples were polished as follows: 220 grit (European standard grit) sandpaper was used to remove the surface layer $(\sim 2 \mathrm{~mm})$ of the sample. This was done to ensure that the image obtained was representative of the sample, and not due to some surface irregularity. The surface was then polished using progressively finer grits, ensuring that the surface damage caused by each grit was removed by the following grit. The finest grit used was 1200 grit. The sample was then polished using $6 \mu \mathrm{m}, 3 \mu \mathrm{m}, 1 \mu \mathrm{m}$ and finally $0.25 \mu \mathrm{m}$ diamond suspension. This was done to prepare a smooth surface for the actual etching process. After polishing, the samples were etched using a solution of 1 part $\mathrm{HNO}_{3}$ to 2 parts $\mathrm{H}_{2} \mathrm{O}$.

To gain a reasonable idea of the three-dimensional nature of the grains, the samples were sectioned and polished in two directions: one perpendicular to the direction of deformation in the SHPB, and one parallel to the direction of deformation. The surface perpendicular to the direction of the deformation is denoted as surface $x$, and the surface parallel to the deformation direction is denoted as $y$ (see figure 2). From now on when referring to a sample, the letters $x$ or $y$ will be added depending on which direction the sample is viewed.

TEM samples were prepared by slicing the cylinders into thin layers perpendicular to their axes using EDM, followed by mechanical thinning, punching, polishing and finally ion milling. Mechanical thinning and polishing were performed using the same techniques used during the polishing stage of the SEM sample preparation, and ion milling was performed using a Gatan Model 961 precision ion polishing system. Samples were examined using Philips EM420 and CM20 microscopes at 100 and $200 \mathrm{kV}$, respectively.

\section{Results and discussion}

The experimental observations made regarding the presence of twins in deformed copper can be roughly divided into two classifications regarding their origin depending on whether the samples were viewed using a SEM or TEM apparatus.

\subsection{Samples viewed using SEM}

Images obtained of the sample grain structures with the SEM are shown in figures 3(a) and (b). These images were used to determine the grain size of each sample. This is of importance because grain size is one of the factors influencing the twinning response of deformed copper (Meyers et al 2001). The grain size determination of the samples was done using the linear least squares also known as the Heyn method (Dehoff and Rhines 1968). The average measured grain sizes are shown in table 1. Due to the mangled structure of the unannealed samples, an accurate calculation of the grain size of the samples could not be made.

The fact that the image quality is influenced by the amount of straining that took place, decreases the ease and accuracy by which grain size measurements can be made. The presence of annealing twins in the samples annealed at higher temperature leads to a smaller average grain size than expected. Annealing twins were observed in all the annealed samples. No sign of twinning could be seen in the unannealed samples. Close up views of some of the twins observed using SEM can be seen in figure 4 .

\subsection{Samples viewed using TEM}

Twins were neither found in the original material nor in the annealed but unstrained material. Examination of the unannealed and strained samples also showed no evidence of 

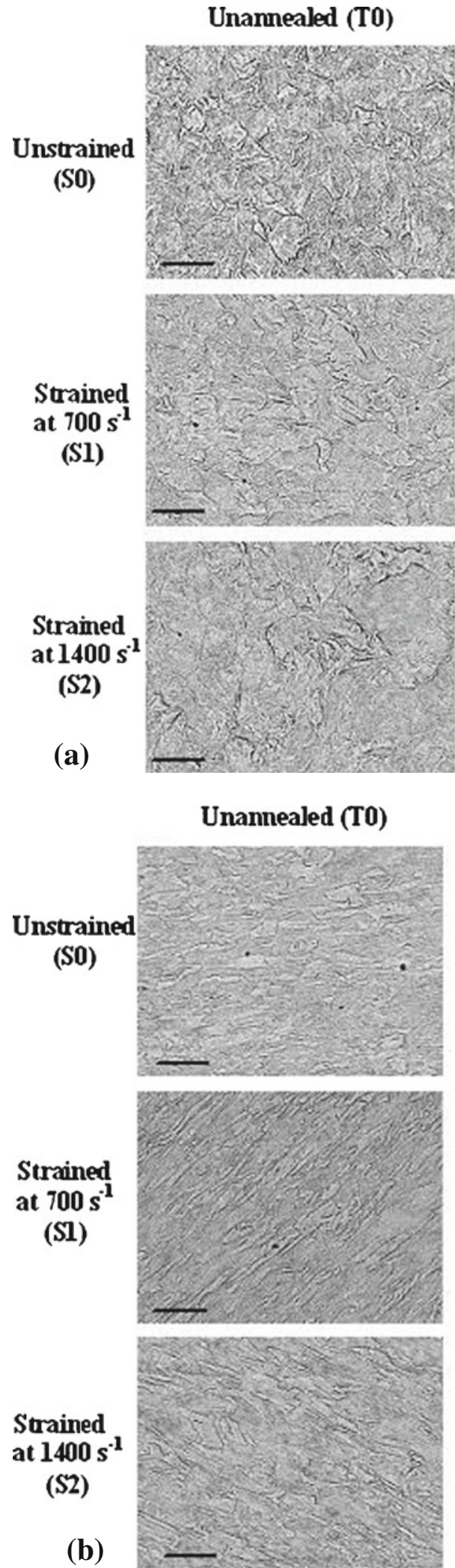

Annealed at $300^{\circ} \mathrm{C}$ (T1)
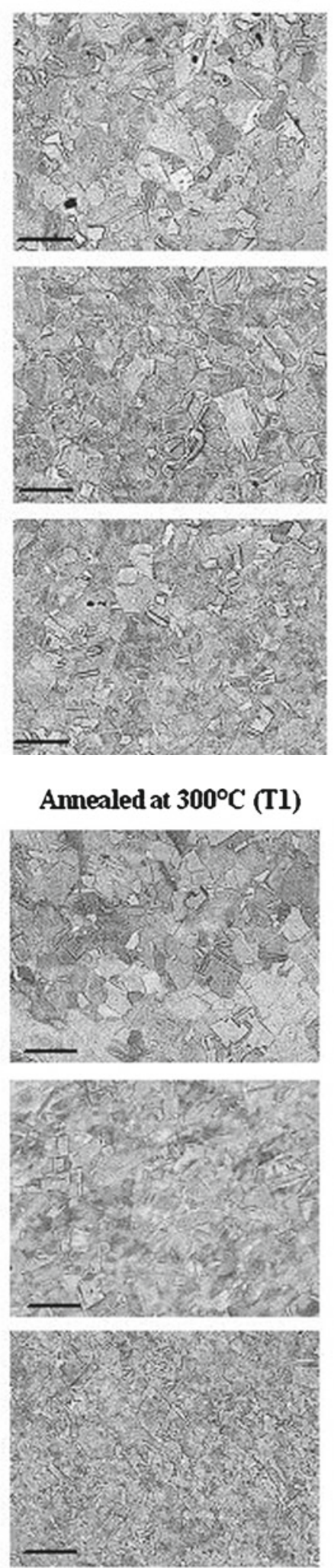

Annealed at $500^{\circ} \mathrm{C}$ (T2)
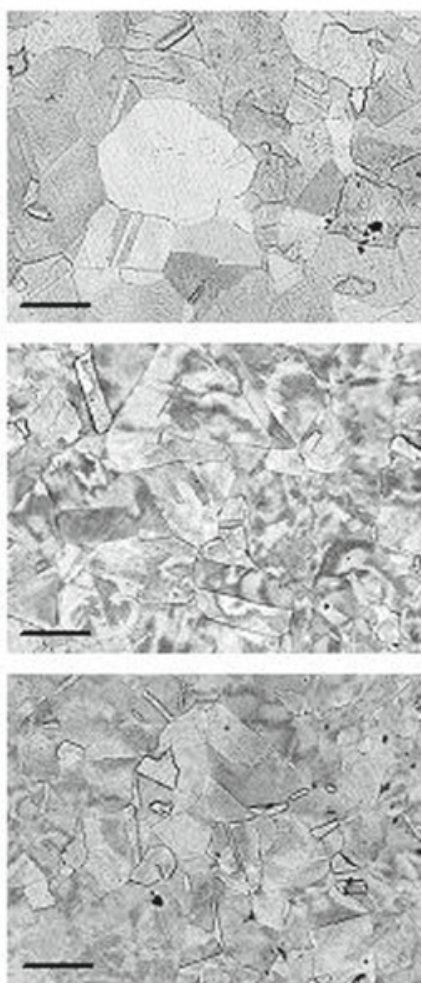

Annealed at $500^{\circ} \mathrm{C}(\mathrm{T} 2)$
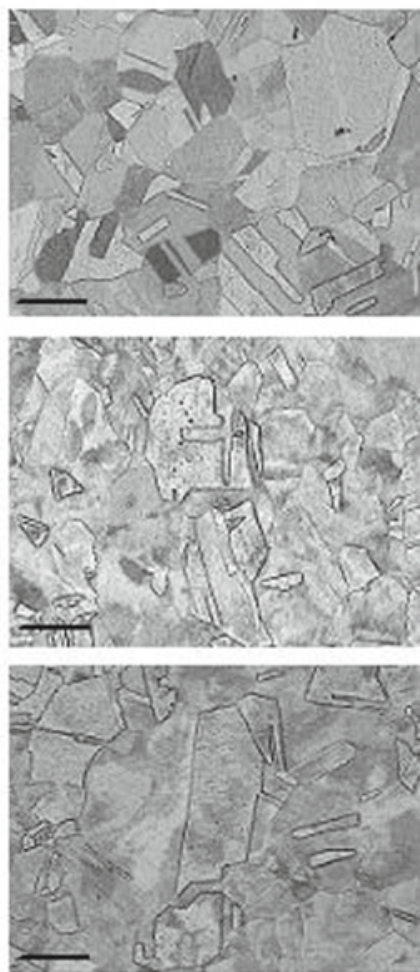

Figure 3. (a) Back scattered electron detector images of copper samples viewed on $x$-surfaces (scale bar is $20 \mu \mathrm{m}$ in each image). (b) Back scattered electron detector images of copper samples viewed on $y$-surfaces (scale bar is $20 \mu \mathrm{m}$ in each image). 
twinning. However, twins were present in all four of the annealed and then strained samples. Figures 5(a) and (b) show the selected area diffraction (SAD) patterns away from, and overlapping, a non-inclined twin and including a twin band in the sample annealed at $500{ }^{\circ} \mathrm{C}$ and strained at $1400 \mathrm{~s}^{-1}$. The first shows many small double diffraction spots due to a surface oxide, while the second contains

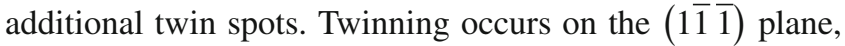
and this diffraction spot almost coincides in the matrix and twinned patterns, with a small misorientation possibly due to many dislocations near the interface (Toa et al 2004). Figure 5(c) shows a bright field (BF) image of the twin, while figure $5(\mathrm{~d})$ is a dark field (DF) image of the same region using a twin spot.

Figure 6(a) shows a series of thin twin bands in the sample annealed at $500{ }^{\circ} \mathrm{C}$ and strained at $700 \mathrm{~s}^{-1}$.

Streaking (due to the planar defect being parallel to the electron beam) in the diffraction pattern perpendicular to these thin bands is evident in the corresponding SAD pattern given in figure 6(b). Figure 7 shows twin band with one twin having a slight misorientation.

Table 1. Average grains size of copper samples calculated using the Heyn method. ( $\mathrm{T} 0=$ unannealed, $\mathrm{T} 1=300{ }^{\circ} \mathrm{C}, \mathrm{T} 2=500{ }^{\circ} \mathrm{C}$, $\mathrm{S} 0=$ unstrained, $\mathrm{S} 1=700 \mathrm{~s}^{-1}, \mathrm{~S} 2=1400 \mathrm{~s}^{-1}$ ).

\begin{tabular}{lcc}
\hline Samples & $\begin{array}{c}\text { Grain size on } \\
X \text { plane }(\mu \mathrm{m})\end{array}$ & $\begin{array}{c}\text { Grain size on } \\
Y \text { plane }(\mu \mathrm{m})\end{array}$ \\
\hline T0S0 & Undetermined & Undetermined \\
T0S1 & Undetermined & Undetermined \\
T0S2 & Undetermined & Undetermined \\
T1S0 & 5 & 5 \\
T1S1 & 5 & 5 \\
T1S2 & 5 & 4 \\
T2S0 & 8 & 7 \\
T2S1 & 9 & 10 \\
T2S2 & 9 & 8 \\
\hline
\end{tabular}

\section{Discussion}

Since twins were only observed using TEM in the strained material, these twins were attributed to deformation rather than annealing. This was unexpected as strain rates required for deformation twinning are much higher (see slip-twin transition in figure 8). Yet deformation twinning of copper does occur when it is deformed at low (liquid nitrogen) temperatures or at high $\left(\sim 1000 \mathrm{~s}^{-1}\right)$ strain rates. Deformation twinning has also been reported in copper undergoing low strain rate, severe plastic deformation at room temperature (Huang et al 2006).

The twins observed using SEM are clearly annealing twins as they do not occur in the unannealed samples. However, the twins observed using TEM in the annealed and strained samples cannot be annealing twins, since they were not observed in the only-annealed samples. They are therefore identified as deformation twins. Furthermore, these deformation twins are in the order of nanometres, whereas the annealing twins observed using SEM were several microns in size.

It is important to note that these twins observed using TEM were a prominent characteristic of the samples in which they were encountered. As an example, almost all of the grains in the sample annealed at $300{ }^{\circ} \mathrm{C}$ and strained at $700 \mathrm{~s}^{-1}$ contained twins. These twin bands, ranging in width from a few nanometers up to approximately $550 \mathrm{~nm}$ constituted up to $5 \%$ of the grain surface viewed.

These deformation twins may have been formed by the motion of partial dislocations called twinning dislocations with $\boldsymbol{b}=a / 6\langle 112\rangle$ on consecutive (111) planes. The movement of these twinning - or transformation dislocations creates changes in orientation or phases. In this case it is believed that their movement or glide created the twins (Williams and Carter 1996). An explanation for the nucleation mechanism of these twins is that of the perfect dislocation pole-model (Sleeswyk 1974). In this model a twin can be obtained if the mechanism is nucleated from a triple node of perfect dislocations. When a stress is applied the two Shockley partials of which one of these dislocations is composed (a)

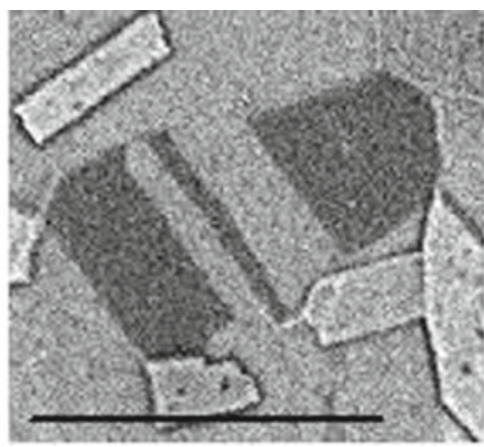

(b)

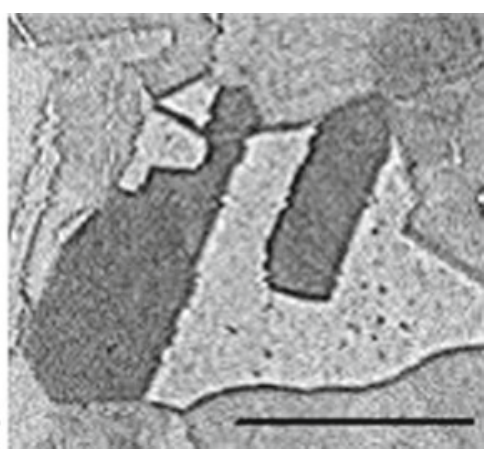

Figure 4. Annealing twins in sample annealed at $500{ }^{\circ} \mathrm{C}$ and strained at $1400 \mathrm{~s}^{-1}$ : (a) type 1 and (b) types 1 and 2 combined as shown in figure 1 . Scale bar indicates $20 \mu \mathrm{m}$. 


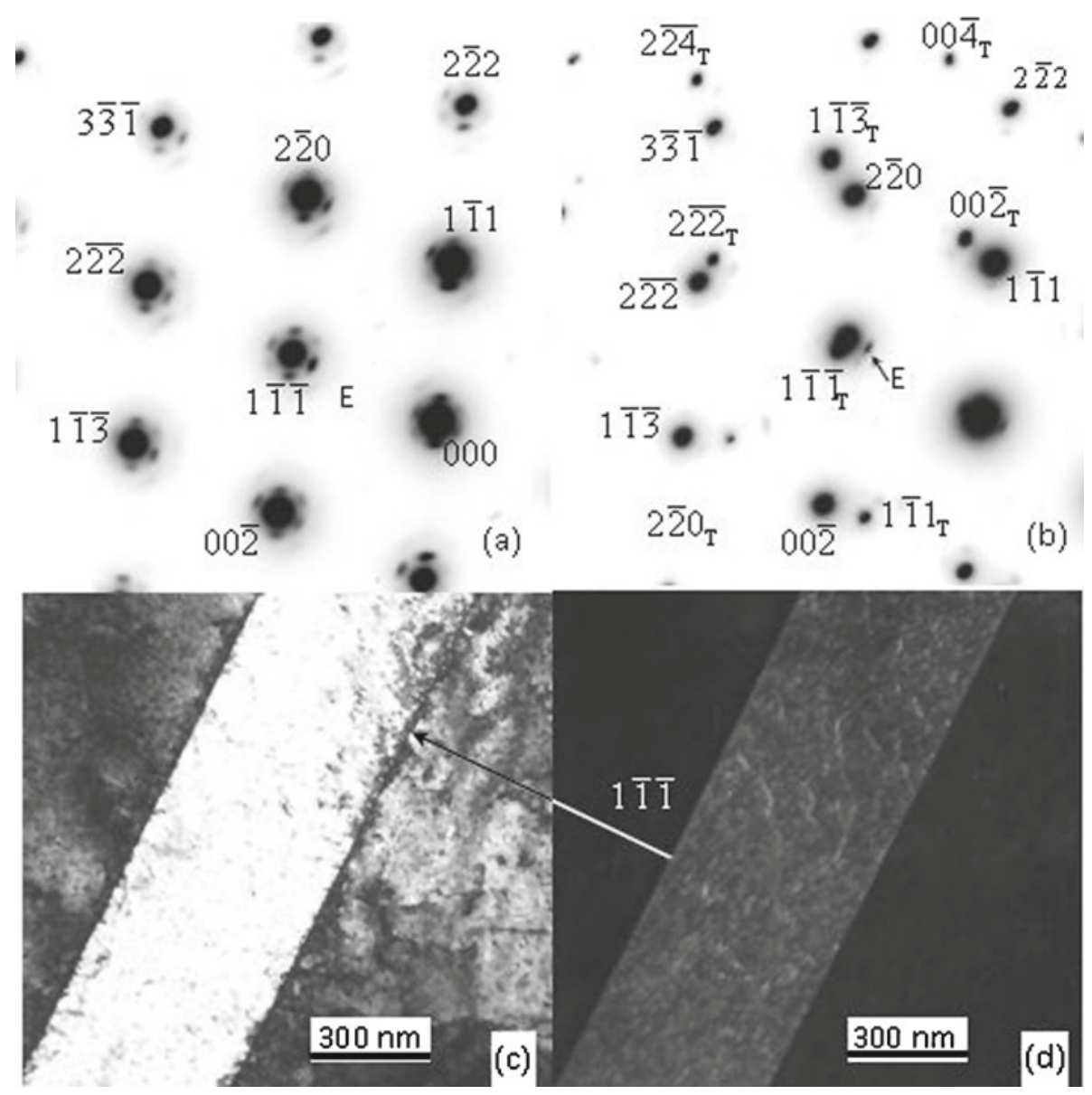

Figure 5. (a) SAD pattern in (110) zone of copper, away from twin, with many small double diffraction spots (such as one labeled E) due to an oxide layer, (b) SAD pattern of region overlapping twin, showing additional twin spots, (c) BF image of twin and (d) DF image of twin using a twin diffraction spot $(200 \mathrm{kV})$.

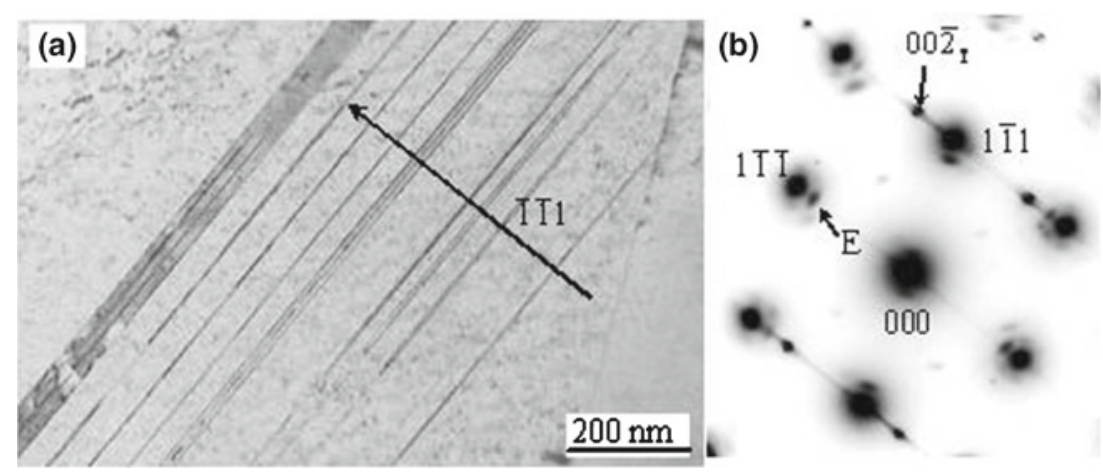

Figure 6. (a) BF image of twin bands and (b) corresponding SAD pattern showing streaks, twin spots and double diffraction spots (e.g. spot E) $(100 \mathrm{kV})$.

may rotate in mutually opposite senses around the other two perfect dislocations, producing a single twin lamella.

These deformation twins formed at a much lower strain rate than predicted by Meyers et al (2001) (see figure 8) for the onset of twinning. Their model, however, is questionable because the stress required for twinning was assumed to be constant, whereas it actually decreases with increasing grain size (Huang et al 2006).

It is interesting that deformation twinning did not occur in the unannealed-strained samples, and this is not fully understood. One possibility is that a larger grain size in the annealed samples reduced the stress required for the onset of 


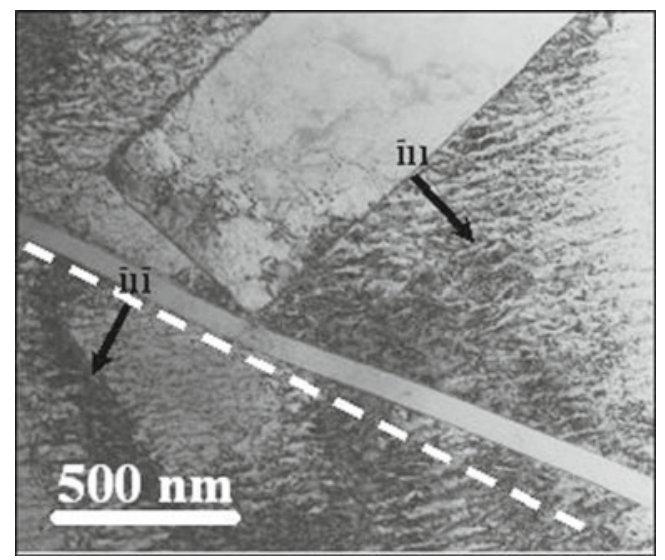

Figure 7. BF image of twin bands in sample annealed at $300{ }^{\circ} \mathrm{C}$ and strained at $700 \mathrm{~s}^{-1}$ showing slight misorientation. $(200 \mathrm{kV})$.

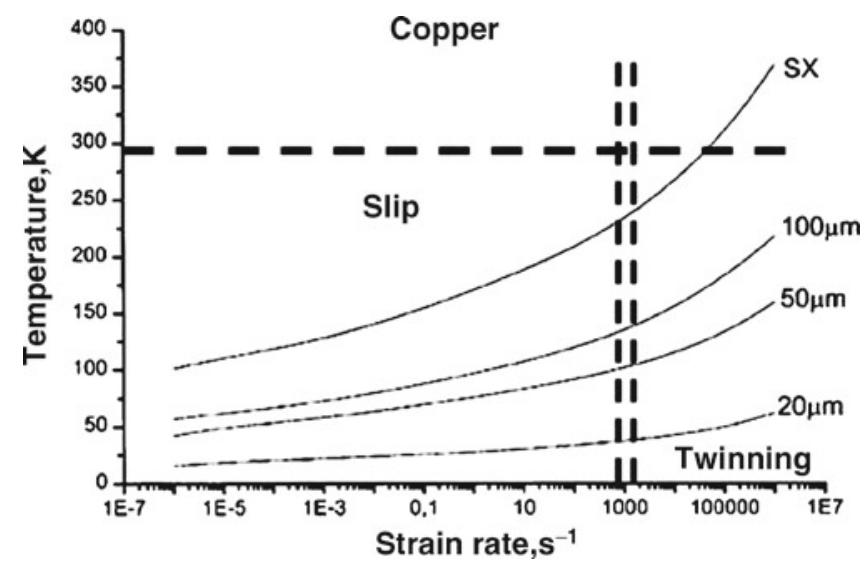

Figure 8. Model of Meyers et al (2001) for slip-twinning threshold in copper. Dashed lines indicate straining conditions used in this study (SX means single crystalline) (Meyers et al 2001).

twinning, which is certainly the case for the higher temperature anneal. A second aspect is that the unannealed samples were strained to only $\sim 7 \%$, whereas the annealed samples were strained to $\sim 14 \%$, making twinning more likely.

\section{Conclusions}

Twins were observed in copper samples using both SEM and TEM. The twins observed using SEM were several microns in size and are annealing twins. However, the twins observed by TEM are believed to have formed during deformation, as no twins were found in the samples that were left unstrained. The unannealed but strained samples also showed no evidence of twinning. However, twins were present in all four of the annealed and then strained samples. The twins found in the annealed and strained samples cannot be annealing twins, since they did not occur in the only-annealed samples, and are therefore identified as deformation twins. Furthermore, these deformation twins are in the order of nanometres in size. These deformation twins formed at a much lower strain rate than predicted by Meyers et al (2001). Due to the predominance of these deformation twins they are considered to be an important observation.

\section{Acknowledgements}

The electron microscope images in this study were obtained at the Centre for confocal and electron microscopy at the University of the Free State as well as at the Department of Physics at the Nelson Mandela Metropolitan University. The assistance of the staff is gratefully acknowledged. The authors gratefully acknowledge the provision of samples and the financial assistance of Dr Jean Terblanche and Denel Land Systems Western Cape. The financial assistance of the National Research Foundation of South Africa in the form of a travel grant is also gratefully acknowledged.

\section{References}

Cullity B D 1978 Elements of X-ray diffraction (Addison-Wesley Publishing Company) p. 67

Dehoff R and Rhines F 1968 Quantitive microscopy (New York: McGraw Hill Ltd.) p. 239

Gray G T 2000 Classic split-Hopkinson pressure bar testing in ASM handbook (eds) H Huhn and D Medlin (Materials Park, Ohio: ASM International) Vol. 8, p. 462

Huang C X, Wang K, Wu S D, Zhang Z F, Li G Y and Li S X 2006 Acta Mater. 54655

Marais S T, Tait R B, Cloete T J and Nurick G N 2004 Lat. Am. J. Solid. Struct. 1319

Meyers M A, Vöhringer O and Lubarda V A 2001 Acta Mater. 49 4025

Sleeswyk A W 1974 Phil. Mag. 29407

Toa N R, Wu X L, Sui M L, Lu J and Lu K 2004 J. Mater. Res. 19 1623

Williams D B and Carter C B 1996 Transmission electron microscopy (New York: Plenum Press) Vol. 3, p. 414 